\title{
NEW $Z$-DOMAIN CONTINUED FRACTION EXPANSIONS BASED ON AN INFINITE NUMBER OF MIRROR-IMAGE AND ANTI-MIRROR-IMAGE POLYNOMIAL DECOMPOSITIONS
}

\author{
V. RAMACHANDRAN \\ Department of Electrical and Computer Engineering, \\ Concordia University, \\ Montreal, Quebec, Canada, HЗG 1 M8 \\ kamala@ece.concordia.ca \\ RAVI P. RAMACHANDRAN \\ Department of Electrical and Computer Engineering, \\ Rowan University, \\ Glassboro, New Jersey, 08028, USA \\ ravi@rowan.edu \\ C. S. GARGOUR \\ Department of Electrical Engineering, \\ Ećole Technologie Superieure, \\ Montreal, Quebec, Canada, H2T 2C8 \\ Christian.Gargour@etsmtl.ca
}

Revised 13 November 2007

\begin{abstract}
A magnitude response preserving modification of the denominator polynomial of a causal and stable digital transfer function leads to an infinite number of decompositions into a mirror-image polynomial (MIP) and an anti-mirror-image polynomial (AMIP). Properties and identifications of the MIP and AMIP are given. The identifications of Schussler and Davis, and the line spectral frequency formulation are special cases of the general MIP and AMIP decompositions introduced in this paper. Two types of Discrete Reactance Functions (DRF) are constructed. From these DRFs, five new continued fraction expansions $(\mathrm{CFE})$ are developed, and some properties are obtained.
\end{abstract}

Keywords: Mirror-image polynomial; anti-mirror-image polynomial; discrete reactance function; continued fraction expansion.

\section{Introduction}

An $n$th order denominator polynomial $D_{n}(z)$ can be decomposed as a sum of a mirror-image polynomial (MIP) and an anti-mirror-image polynomial (AMIP). One of the decompositions is due to Schussler. ${ }^{1,2}$ This is used in the implementation of a stability test. ${ }^{3}$ Another decomposition is due to Davis, ${ }^{4}$ which can also be used to 
determine the stability of the system. ${ }^{5}$ In this paper, it is shown that several other new possibilities of decomposing $z^{q} D_{n}(z)$ into a sum of a MIP and an AMIP exist, and some of the properties of such polynomials are discussed. Two new types of Discrete Reactance Functions (DRF) are constructed and new continued fraction expansions (CFE) are derived.

\section{Mirror-Image and Anti-Mirror-Image Polynomials}

Let

$$
D_{n}(z)=\sum_{i=0}^{n} d(i) z^{i}
$$

be a minimum phase polynomial. One can formulate a stable all-pass transfer function given by

$$
H_{n+q}(z)=\frac{z^{n} D_{n}\left(z^{-1}\right)}{z^{q} D_{n}(z)},
$$

where $q \geq 0$, and $n+q$ is the order of $H_{n+q}(z)$. Based on $D_{n}(z)$, we can now define an MIP, $M_{n+q}(z)$ and an AMIP, $A_{n+q}(z)$ as follows:

$$
\begin{aligned}
M_{n+q}(z) & =\frac{1}{2}\left[z^{q} D_{n}(z)+z^{n} D_{n}\left(z^{-1}\right)\right], \\
A_{n+q}(z) & =\frac{1}{2}\left[z^{q} D_{n}(z)-z^{n} D_{n}\left(z^{-1}\right)\right] .
\end{aligned}
$$

In general, a polynomial of order $n$, namely, $P_{n}(z)$ is an MIP if $P_{n}(z)=z^{n} P_{n}\left(z^{-1}\right)$. Similarly, $P_{n}(z)$ is an AMIP if $P_{n}(z)=-z^{n} P_{n}\left(z^{-1}\right)$. Obviously, $M_{n+q}(z)$ is an $(n+q)$ th order polynomial obtained as half the sum of the denominator and the numerator polynomials of $H_{n+q}(z)$. Similarly, $A_{n+q}(z)$ is an $(n+q)$ th order polynomial obtained as half the difference of the denominator and the numerator polynomials of $H_{n+q}(z)$. It is clear that, depending on the value of $q$ chosen, an infinite number of pairs of MIPs and AMIPs is obtained. Reconstruction of $D_{n}(z)$ is possible as

$$
D_{n}(z)=z^{-q}\left(M_{n+q}(z)+A_{n+q}(z)\right)
$$

We will now consider some properties of these polynomials.

\section{Properties and Identifications of MIP and AMIP}

First, we have introduced a novel general MIP and AMIP identification given in Eqs. (3) and (4). Note that in the sequel, $M_{s}(z)$ denotes an MIP of order $s$ and 
$A_{s}(z)$ denotes an AMIP of order $s$. The general factorization properties of $M_{n+q}(z)$ and $A_{n+q}(z)$ are given as follows:

Case 1. Suppose $|q-n|$ is odd. Since $(z+1)$ is only a factor of $M_{n+q}(z)$ and $(z-1)$ is only a factor of $A_{n+q}(z)$, one can write

$$
\begin{aligned}
& M_{n+q}(z)=(z+1) M_{n+q-1}^{(-1)}(z), \\
& A_{n+q}(z)=(z-1) M_{n+q-1}^{(1)}(z),
\end{aligned}
$$

where $M_{n+q-1}^{(-1)}(z)$ and $M_{n+q-1}^{(1)}(z)$ are different MIPs of order $n+q-1$. The superscript $(-1)$ indicates that the MIP was derived from a polynomial of higher order with the root at $z=-1$ removed or deconvolved. Similarly, the superscript 1 indicates that the MIP was derived from a polynomial of higher order with the root at $z=1$ removed or deconvolved.

Case 2. Suppose $|q-n|$ is even. Since $(z+1)$ and $(z-1)$ are only factors of $A_{n+q}(z)$, one can write

$$
\begin{aligned}
A_{n+q}(z) & =(z-1) M_{n+q-1}^{(1)}(z) \\
& =(z+1) A_{n+q-1}^{(-1)}(z),
\end{aligned}
$$

where $M_{n+q-1}^{(1)}(z)$ is an MIP of order $n+q-1$ and $A_{n+q-1}^{(-1)}(z)$ is an AMIP of order $n+q-1$. Roots at $z= \pm 1$ are appropriately deconvolved.

The new identification and general factorization properties we give above have special cases. First, consider $q=0$. For $n$ odd, the factorization given in Case 1 above holds in that

$$
\begin{aligned}
M_{n}(z) & =\frac{1}{2}\left[D_{n}(z)+z^{n} D_{n}\left(z^{-1}\right)\right] \\
& =(z+1) M_{n-1}^{(-1)}(z)
\end{aligned}
$$

and

$$
\begin{aligned}
A_{n}(z) & =\frac{1}{2}\left[D_{n}(z)-z^{n} D_{n}\left(z^{-1}\right)\right] \\
& =(z-1) M_{n-1}^{(1)}(z) .
\end{aligned}
$$

Similarly, for $n$ even, the factorization given in Case 2 above holds in that

$$
\begin{aligned}
A_{n}(z) & =\frac{1}{2}\left[D_{n}(z)-z^{n} D_{n}\left(z^{-1}\right)\right] \\
& =(z-1) M_{n-1}^{(1)}(z) \\
& =(z+1) A_{n-1}^{(-1)}(z) .
\end{aligned}
$$


The special case $q=0$ is Schussler's identification. ${ }^{1}$ Reconstruction is possible using $D_{n}(z)=M_{n}(z)+A_{n}(z)$. For $D_{n}(z)$ to be of minimum phase, it is necessary and sufficient that (1) $|d(n)|>|d(0)|$ and (2) the roots of the MIP $M_{n}(z)$ and the AMIP $A_{n}(z)$ are on the unit circle, simple and interlace. ${ }^{1,2}$ This has been further implemented as a part of a stability test $^{3}$ and a subsequent formulation for the design of optimum least-squares infinite impulse response filters in which stability is guaranteed. ${ }^{6}$

Another special case arises when $q=1$. For $n$ even and $n$ odd, the factorizations given in Cases 1 and 2, respectively, are applicable. For even $n$,

$$
\begin{aligned}
M_{n+1}(z) & =\frac{1}{2}\left[z D_{n}(z)+z^{n} D_{n}\left(z^{-1}\right)\right] \\
& =(z+1) M_{n}^{(-1)}(z)
\end{aligned}
$$

and

$$
\begin{aligned}
A_{n+1}(z) & =\frac{1}{2}\left[z D_{n}(z)-z^{n} D_{n}\left(z^{-1}\right)\right] \\
& =(z-1) M_{n}^{(1)}(z)
\end{aligned}
$$

For $n$ odd,

$$
\begin{aligned}
A_{n+1}(z) & =\frac{1}{2}\left[z D_{n}(z)-z^{n} D_{n}\left(z^{-1}\right)\right] \\
& =(z-1) M_{n}^{(1)}(z) \\
& =(z+1) A_{n}^{(-1)}(z) .
\end{aligned}
$$

Reconstruction is possible using $D_{n}(z)=z^{-1}\left(M_{n+1}(z)+A_{n+1}(z)\right)$. Again, for a minimum phase $D_{n}(z)$, it is necessary and sufficient that the roots of the MIP $M_{n+1}(z)$ and the AMIP $A_{n+1}(z)$ are on the unit circle, simple and interlace. This special case is the line spectral frequency (LSF) formulation commonly used in speech processing. The LSFs were first introduced by Itakura $^{7}$ as the angular frequencies of the unit circle roots of the $\operatorname{MIP} M_{n+1}(z)$ and the $\operatorname{AMIP} A_{n+1}(z)$ neglecting the roots at $z= \pm 1$. The polynomial $D_{n}(z)$ is derived by linear predictive analysis. ${ }^{8}$ The LSFs are commonly used in speech coding due to their intimate relationship with the speech spectral envelope thereby making them conducive for transmission at low bit rates. ${ }^{9-11}$

The case $q=1$ has also resulted in Davis' identification of $D_{n}(z)$ as the sum of an MIP and an AMIP of different orders derived from the Schussler and LSF formulations. ${ }^{4}$ This has also been implemented as a part of a stability test. ${ }^{5}$ For even $n$, Davis' identification is

$$
D_{n}(z)=M_{n}^{(-1)}(z)+A_{n-1}^{(-1)}(z),
$$


where $M_{n}^{(-1)}(z)$ is defined in Eq. (18) and $A_{n-1}^{(-1)}(z)$ is defined in Eq. (16). For odd $n$, Davis' identification is

$$
D_{n}(z)=M_{n-1}^{(-1)}(z)+A_{n}^{(-1)}(z)
$$

where $M_{n-1}^{(-1)}(z)$ is defined in Eq. (11) and $A_{n}^{(-1)}(z)$ is defined in Eq. (23).

\section{Continued Fraction Expansions}

Here, we introduce new results based on $M_{n+q}(z)$ and $A_{n+q}(z)$ defined in Eqs. (3) and (4).

Theorem 1. The magnitude of the all-pass function $H_{n+q}(z)$ (see Eq. (2)) on various circles in the z-plane of different radii $r$ is given as

$$
\left|H_{n+q}\left(r e^{j \omega}\right)\right| \text { is } \begin{cases}>1 & \text { for } r<1, \\ =1 & \text { for } r=1, \\ <1 & \text { for } r>1,\end{cases}
$$

The proof of this theorem is in Ref. 12. The result of Theorem 1 leads to Theorem 2.

Theorem 2. Let

$$
R_{n+q}(z)=\frac{M_{n+q}(z)}{A_{n+q}(z)}
$$

Then,

$$
\operatorname{Re}\left(R_{n+q}\left(r e^{j \omega}\right)\right) \text { is } \begin{cases}<0 & \text { for } r<1 \\ =0 & \text { for } r=1 \\ >0 & \text { for } r>1\end{cases}
$$

where $\operatorname{Re}()$ denotes the real part.

The proof of Theorem 2 is well known. Theorem 2 shows that $R_{n+q}(z)$ is a Positive Exterior Function (PEF). Therefore, $R_{n+q}(z)$ contains all its poles and zeros on the unit circle, which are simple and interlace. ${ }^{13}$ The PEF $R_{n+q}(z)$ and its reciprocal $1 / R_{n+q}(z)$ are Discrete Reactance Functions (DRFs). This permits us to define two types of DRFs, namely,

(1) Type A: The degrees of the numerator and the denominator polynomials are the same. The DRFs are $M_{n+q}(z) / A_{n+q}(z)$ and its reciprocal for various values of $q \geq 0$.

(2) Type B: The degree difference between the numerator and the denominator polynomials is 1 . For any $q \geq 1$, the various DRFs are $M_{n+q}(z) / A_{n+q-1}(z)$, $M_{n+q}(z) / A_{n+q+1}(z), \quad M_{n+q+1}(z) / A_{n+q}(z), \quad M_{n+q-1}(z) / A_{n+q}(z)$, and their reciprocals. 
Each type of DRF can be expanded into continued fractions (CFEs).

Theorem 3. Type A DRF permits the following forms of CFEs: CFE1:

$$
a_{1} \frac{z-1}{z+1}+\frac{1}{a_{2} \frac{z-1}{z+1}+\frac{1}{a_{3} \frac{z-1}{z+1}+\frac{1}{\cdots a_{n+q} \frac{z-1}{z+1}}}}
$$

CFE 2:

$$
b_{1} \frac{z+1}{z-1}+\frac{1}{b_{2} \frac{z+1}{z-1}+\frac{1}{b_{3} \frac{z+1}{z-1}+\frac{1}{\cdots b_{n+q} \frac{z+1}{z-1}}}} .
$$

CFE3: When $n+q$ is even, we get

$$
c_{1} \frac{z+1}{z-1}+c_{2} \frac{z-1}{z+1}+\frac{1}{c_{3} \frac{z+1}{z-1}+c_{4} \frac{z-1}{z+1}+\frac{1}{\cdots c_{n+q-1} \frac{z+1}{z-1}+c_{n+q} \frac{z-1}{z+1}}} .
$$

CFE4: When $n+q$ is odd, we get

$$
d_{0} \frac{z+1}{z-1}+\frac{1}{d_{1} \frac{z+1}{z-1}+d_{2} \frac{z-1}{z+1}+\frac{1}{\cdots d_{n+q-2} \frac{z+1}{z-1}+d_{n+q-1} \frac{z-1}{z+1}}} .
$$

Proof for CFE1. For CFE1, there are two cases to be considered. The first case is when $|q-n|$ is even. Then, $(z+1)$ is a factor of $A_{n+q}(z)$, and

$$
A_{n+q}(z)=(z+1) A_{n+q-1}^{(-1)}(z) .
$$

Therefore, one can write

$$
\frac{M_{n+q}(z)}{A_{n+q}(z)}=a_{1} \frac{z-1}{z+1}+\psi_{1}(z),
$$

where the denominator of $\psi_{1}(z)$ is $A_{n+q-1}^{(-1)}(z)$. It has been shown in Ref. 14 that $a_{1}>0$ and $\psi_{1}(z)$ is a PEF of odd order. Also, the numerator of $\psi_{1}(z)$ contains $(z+1)$ as a factor. Therefore,

$$
\frac{1}{\psi_{1}(z)}=a_{2} \frac{z-1}{z+1}+\psi_{2}(z)
$$

Continuing this process establishes CFE1. 
The second case for CFE1 is when $|q-n|$ is odd. Then, $(z+1)$ is only a factor of $M_{n+q}(z)$ and $(z-1)$ is only a factor of $A_{n+q}(z)$. Therefore, one can write

$$
\frac{M_{n+q}(z)}{A_{n+q}(z)}=(0) \frac{z-1}{z+1}+\frac{1}{A_{n+q}(z) / M_{n+q}(z)} .
$$

Since $(z+1)$ is a factor of $M_{n+q}(z)$, one can write

$$
\frac{M_{n+q}(z)}{A_{n+q}(z)}=(0) \frac{z-1}{z+1}+\frac{1}{a_{2} \frac{z-1}{z+1}+\psi_{2}(z)} .
$$

The expansion of CFE1 results by continuing this process.

Proof for CFE2. For CFE2, there are also two cases to be considered. The first case is when $|q-n|$ is even. Then, $(z-1)$ is a factor of $A_{n+q}(z)$, and one can write

$$
\frac{M_{n+q}(z)}{A_{n+q}(z)}=b_{1} \frac{z+1}{z-1}+\phi_{1}(z),
$$

where the denominator of $\phi_{1}(z)$ is $M_{n+q-1}^{(-1)}(z)$. It has been shown in Ref. 14 that $b_{1}>0$ and $\phi_{1}(z)$ is a PEF of odd order. Also, the numerator of $\phi_{1}(z)$ contains $(z-1)$ as a factor. Therefore,

$$
\frac{1}{\phi_{1}(z)}=b_{2} \frac{z+1}{z-1}+\phi_{2}(z)
$$

Continuing this process establishes CFE2.

If $|q-n|$ is even, $(z-1)$ is again a factor of $A_{n+q}(z)$. The proof of CFE2 follows along the same lines as for the case when $|q-n|$ is odd. For CFE2, the values of $b_{1}, b_{2}, \ldots$ always exist if one starts with $M_{n+q}(z) / A_{n+q}(z)$ and not its reciprocal.

Proof for CFE3. For CFE3, $|q-n|$ is even and both $(z-1)$ and $(z+1)$ are factors of $A_{n+q}(z)$. Therefore, one can write

$$
\frac{M_{n+q}(z)}{A_{n+q}(z)}=c_{1} \frac{z+1}{z-1}+c_{2} \frac{z-1}{z+1}+\lambda_{1}(z) .
$$

From the knowledge of CFE1 and CFE2, it is seen that $c_{1}>0, c_{2}>0$, and $\lambda_{1}(z)$ is a PEF of even order. Also, the numerator of $\lambda_{1}(z)$ contains both $(z-1)$ and $(z+1)$ as factors. Therefore,

$$
\frac{1}{\lambda_{1}(z)}=c_{3} \frac{z+1}{z-1}+c_{4} \frac{z-1}{z+1}+\lambda_{2}(z) .
$$

Continuing this process establishes CFE3. 
Proof for CFE4. For CFE4, $|q-n|$ is odd and $(z-1)$ is a factor of $A_{n+q}(z)$. Therefore, one can write

$$
\frac{M_{n+q}(z)}{A_{n+q}(z)}=d_{0} \frac{z+1}{z-1}+\mu_{1}(z) .
$$

From the knowledge of CFE1 and CFE2, it is seen that $d_{0}>0$, and $\mu_{1}(z)$ is a PEF of even order. Also, the numerator of $\mu_{1}(z)$ contains both $(z-1)$ and $(z+1)$ as factors. Therefore,

$$
\frac{1}{\mu_{1}(z)}=d_{1} \frac{z+1}{z-1}+d_{2} \frac{z-1}{z+1}+\mu_{2}(z) .
$$

Continuing this process establishes CFE4.

The expansions CFE1 and CFE2 have been indicated in Ref. 14 for the special case $q=0$. Stability of $1 / D_{n}(z)$ is ensured when each of the set of coefficients $a_{i}, b_{i}$, and either of $c_{i}$ or $d_{i}$ is positive. Each individual set of coefficients provide the necessary and sufficient conditions for stability. Hence, any one CFE can be used. Note also that some of the coefficients may not exist. For example, if $z+1$ is not a factor of the denominator polynomial of the Type A DRF, $a_{1}$ and $c_{2}$ will not exist. This can be seen for the example depicted in Table 1. In this situation, the CFE can still be considered or the CFE of the reciprocal can be considered. Similarly, $z-1$ may not be a factor of the denominator polynomial of the Type A $\mathrm{DRF} A_{n+q}(z) / M_{n+q}(z)$. In this case, $b_{1}, c_{1}$, and $d_{0}$ will not exist. Again, the CFE can still be considered or the CFE of the reciprocal can be considered. Whether the first coefficient exists or not, the number of coefficients in the CFE will still be equal to the order of the MIP or AMIP.

Theorem 4. Type B DRF permits the following form of CFE: CFE5:

$$
e_{1}(z-1)+\frac{1}{e_{2}\left(1-z^{-1}\right)+\frac{1}{e_{3}(z-1)+\frac{1}{e_{4}\left(1-z^{-1}\right)+\cdots}}} .
$$

The proof is similar to the one given in Ref. 4 and is omitted for the sake of brevity. For the case $q=1$, the coefficients correspond to those given in Ref. 4 . The number of coefficients in CFE5 is equal to the order of the MIP or AMIP, whichever is higher. If there is a common factor between the MIP and AMIP, the number of coefficients of CFE5 reduces by one. The set of coefficients $e_{i}$ being positive provides only a necessary condition for the stability of $1 / D_{n}(z)$.

Numerical Example. Consider the minimum phase polynomial $D_{3}(z)=3 z^{3}+$ $2 z^{2}+z+1$. For $q=0$, the MIP and AMIP are given by

$$
\begin{aligned}
& 2 M_{3}(z)=4 z^{3}+3 z^{2}+3 z+4 \\
& 2 A_{3}(z)=2 z^{3}+z^{2}-z-2 .
\end{aligned}
$$


For $q=1$, the MIP and AMIP are given by

$$
\begin{aligned}
2 M_{4}(z) & =3 z^{4}+3 z^{3}+2 z^{2}+3 z+3, \\
2 A_{4}(z) & =3 z^{4}+z^{3}-z-3 .
\end{aligned}
$$

For $q=2$, the MIP and AMIP are given by

$$
\begin{aligned}
& 2 M_{5}(z)=3 z^{5}+2 z^{4}+2 z^{3}+2 z^{2}+2 z+3, \\
& 2 A_{5}(z)=3 z^{5}+2 z^{4}-2 z-3 .
\end{aligned}
$$

Figure 1 shows the roots of $D_{3}(z), M_{5}(z)$, and $A_{5}(z)$. Note how the roots of $A_{5}(z)$ and $M_{5}(z)$ interlace on the unit circle.

The coefficients of the CFEs for the DRFs of Type A are given in Tables 1 and 2. It is readily observed that all the coefficients of the CFEs are positive, thereby showing that the roots of $D_{3}(z)$ are contained within the unit circle. Note also that since $(z+1)$ is not a factor of $A_{3}(z)$ and $A_{5}(z), a_{1}$ does not exist in either case. However, the number of CFE coefficients is still equal to the order of the MIP or AMIP.

The coefficients of CFE5 for the DRF of Type B are given in Table 3. It is readily observed that all the coefficients of the CFE are positive which is a necessary condition for the roots of $D_{3}(z)$ to be within the unit circle. Note also that $A_{4}(z) / M_{3}(z)$

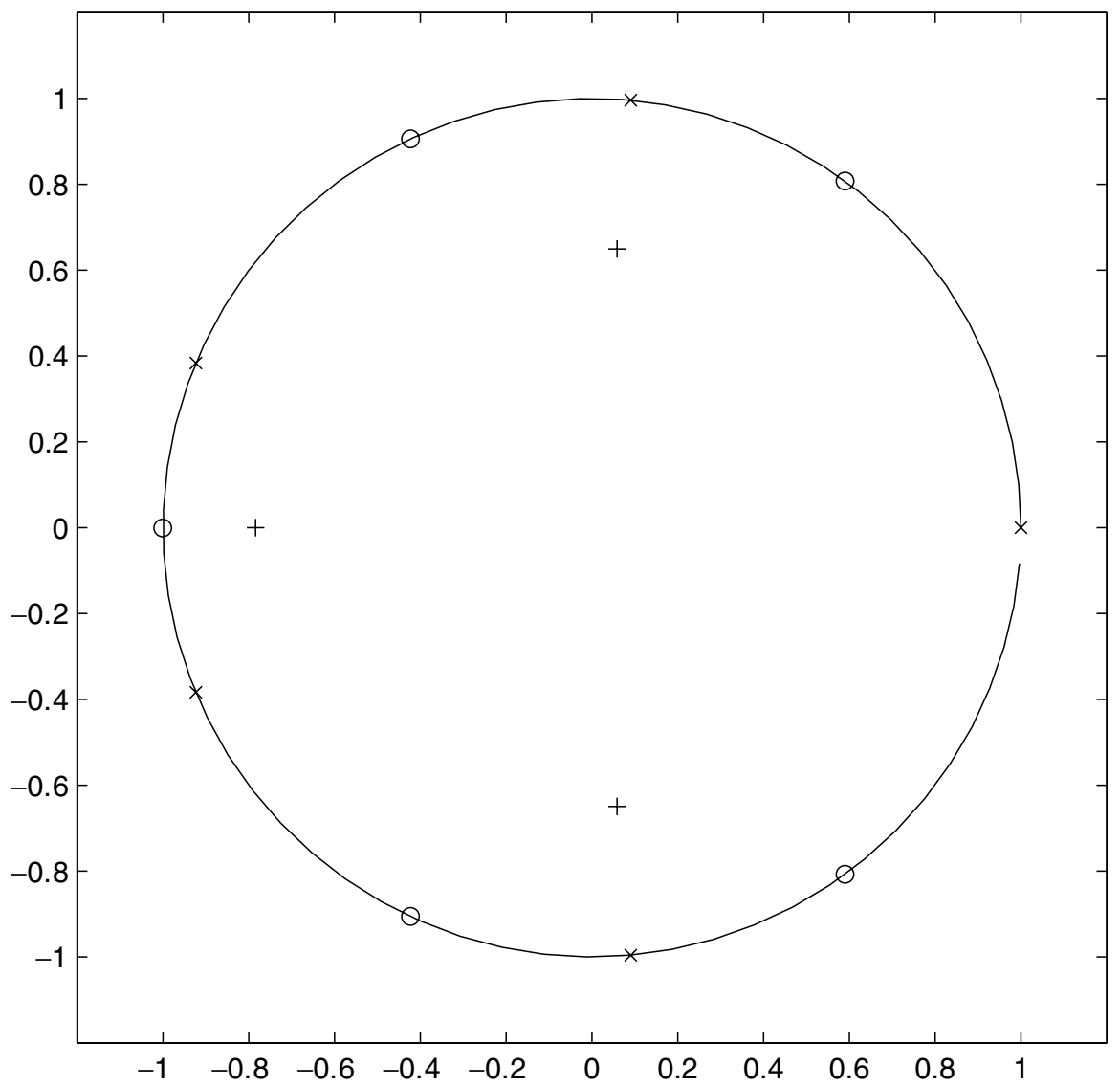

Fig. 1. Roots of $D_{3}(z)$ (denoted by a + ), $M_{5}(z)$ (denoted by a $\bigcirc$ ), and $A_{5}(z)$ (denoted by an $\left.X\right)$. 
Table 1. Coefficients of CFE1 and CFE2 for three different Type A DRFs corresponding to $q=0,1$, and 2 .

\begin{tabular}{cll}
\hline DRF & \multicolumn{1}{c}{ CFE1 } & CFE2 \\
\hline$M_{3}(z) / A_{3}(z)$ & \multicolumn{1}{c}{$a_{1}$ does not exist } & $b_{1}=1$ \\
& $a_{2}=1 / 9$ & $b_{2}=7 / 8$ \\
& $a_{3}=81 / 56$ & $b_{3}=8$ \\
& $a_{4}=56 / 63$ & \\
$M_{4}(z) / A_{4}(z)$ & $a_{1}=1 / 10$ & $b_{1}=1 / 2$ \\
& $a_{2}=50 / 73$ & $b_{2}=14 / 11$ \\
& $a_{3}=1387 / 840$ & $b_{3}=121 / 96$ \\
& $a_{4}=336 / 511$ & $b_{4}=96 / 11$ \\
$M_{5}(z) / A_{5}(z)$ & $a_{1}$ does not exist & $b_{1}=1 / 3$ \\
& $a_{2}=1 / 11$ & $b_{2}=63 / 64$ \\
& $a_{3}=121 / 256$ & $b_{3}=128 / 93$ \\
& $a_{4}=2048 / 1793$ & $b_{4}=961 / 576$ \\
& $a_{5}=3214849 / 1951488$ & $b_{5}=576 / 62$ \\
& $a_{6}=243936 / 138061$ & \\
\hline
\end{tabular}

Table 2. Coefficients of CFE3 or CFE4 for three different Type A DRFs corresponding to $q=0,1$, and 2 .

\begin{tabular}{cl}
\hline \multicolumn{1}{c}{$\mathrm{DRF}$} & \multicolumn{1}{c}{ CFE3 or CFE4 } \\
\hline$M_{3}(z) / A_{3}(z)$ & $d_{0}=1, d_{1}=7 / 8, d_{2}=1 / 8$ \\
$M_{4}(z) / A_{4}(z)$ & $c_{1}=1 / 2, c_{2}=1 / 11$, \\
$c_{3}=143 / 108, c_{4}=121 / 108$ \\
$M_{5}(z) / A_{5}(z)$ & $d_{0}=1 / 3, d_{1}=63 / 64, d_{2}=3 / 32$ \\
& $d_{3}=128 / 81, d_{4}=64 / 81$ \\
\hline
\end{tabular}

Table 3. Coefficients of CFE5 for the Type B DRF.

\begin{tabular}{cl}
\hline \multicolumn{1}{c}{$\mathrm{DRF}$} & \multicolumn{1}{c}{ CFE5 } \\
\hline$A_{4}(z) / M_{3}(z)$ & $e_{1}=3 / 4, e_{2}=16 / 7, e_{3}=1 / 4$ \\
$M_{4}(z) / A_{3}(z)$ & $e_{1}=2 / 3, e_{2}=4 / 9, e_{3}=81 / 14$, \\
& $e_{4}=49 / 963$ \\
$M_{5}(z) / A_{4}(z)$ & $e_{1}=1, e_{2}=3 / 4, e_{3}=16 / 7$, \\
& $e_{4}=1 / 4$ \\
$A_{5}(z) / M_{4}(z)$ & $e_{1}=1, e_{2}=3 / 2, e_{3}=4 / 9$, \\
& $e_{4}=81 / 14, e_{5}=49 / 963$ \\
\hline
\end{tabular}

and $M_{5}(z) / A_{4}(z)$ each have a common factor of $(z+1)$ thereby reducing the number of CFE coefficients by one.

Theorem 5. The MIPs and AMIPs defined in Eqs. (3) and (4) have the following properties:
(1) $2 M_{n+q+1}(z)=(z+1) M_{n+q}(z)+(z-1) A_{n+q}(z)$,
(2) $2 A_{n+q+1}(z)=(z-1) M_{n+q}(z)+(z+1) A_{n+q}(z)$,
(3) $2\left[M_{n+q+1}(z)+A_{n+q}(z)\right]=z^{q-1}(z+1) D_{n}(z)$. 
This allows us to easily calculate the MIPs and AMIPs of higher order from the lower order MIPs and AMIPs.

Proof. The proof is by simple algebraic manipulation and hence, only the first property is illustrated.

$$
\begin{aligned}
(z+1) M_{n+q}(z)+(z-1) A_{n+q}(z)= & \frac{1}{2}\left[(z+1) z^{q} D_{n}(z)+(z+1) z^{n} D_{n}\left(z^{-1}\right)\right. \\
& \left.+(z-1) z^{q} D_{n}(z)-(z-1) z^{n} D_{n}\left(z^{-1}\right)\right] \\
= & \frac{1}{2}\left[2 z^{q+1} D_{n}(z)+2 z^{n} D_{n}\left(z^{-1}\right)\right] \\
= & 2 M_{n+q+1}(z) .
\end{aligned}
$$

\section{Summary and Conclusions}

It is shown in this paper that a minimum phase polynomial of the type $z^{q} D_{n}(z)$ can be decomposed into a sum of an MIP and an AMIP. For each value of $q$, there exists an MIP and AMIP pair whose roots on the unit circle, are simple and interlace. The value $q=0$ corresponds to Schussler's identification. The value $q=1$ corresponds to the LSF formulation and Davis' identification. These are particular cases of the new general MIP and AMIP decomposition presented here. From the various MIPs and AMIPs obtained, two types of Discrete Reactance Functions (DRFs) are constructed. From the DRFs, new Continued Fraction Expansions (CFE) are derived that are different from those developed in the related works. ${ }^{5,14-17}$ The above treatment is not possible in the analog domain.

\section{References}

1. H. W. Schussler, A stability theorem for discrete systems, IEEE Trans. Acoust. Speech Signal Process. 24 (1976) 87-89.

2. R. Gnanasekaran, A note on the new 1-D and 2-D stability theorems for discrete systems, IEEE Trans. Acoust. Speech Signal Process. 29 (1981) 1211-1212.

3. V. Ramachandran and C. S. Gargour, Implementation of a stability test based on Schussler's theorem and some consequent coefficient conditions, J. Franklin Instit. 317 (1984) 341-358.

4. A. M. Davis, A new Z-domain continued fraction expansion, IEEE Trans. Circuits Syst. CAS-29 (1982) 658-662.

5. C. Hwang, J.-Hwang and S.-Y. Tsay, A stability test for discrete systems using Davis' $z$-domain continued fraction expansion, IEEE Trans. Circuits Syst.-I: Fund. Theor. Appl. 46 (1999) 1012-1018.

6. V. Ramachandran, C. S. Gargour, M. Ahmadi and M. Borai, Direct design of recursive digital filters based on a new stability test, J. Franklin Instit. 318 (1984) 407-413.

7. F. Itakura, Line spectrum representation of linear predictor coefficients of speech signals, J. Acoust. Soc. Amer. 57 (1975) S35(A).

8. L. R. Rabiner and R. W. Schafer, Digital Processing of Speech Signals (Prentice-Hall, 1978). 
9. N. Sugamara and N. Farvardin, Quantizer design in speech analysis-synthesis, IEEE J. Selected Areas Commun. 6 (1988) 432-440.

10. P. Kroon and K. Swaminathan, A high-quality multirate real-time CELP coder, IEEE J. Selected Areas Commun. 10 (1992) 850-857.

11. R. P. Ramachandran, M. M. Sondhi, N. Seshadri and B. S. Atal, A two codebook format for robust quantization of line spectral frequencies, IEEE Trans. Speech Audio Process. 3 (1995) 157-168.

12. P. P. Vaidyanathan, Multirate Systems and Filter Banks (McGraw-Hill Inc., 1993).

13. A. M. Davis, Realizability-preserving transformations for digital and analog filters, J. Franklin Instit. 311 (1981) 111-121.

14. J. Szczupak, S. K. Mitra and E. I. Jury, Some new results on discrete system stability, IEEE Trans. Acoust. Speech Signal Process. 25 (1977) 101-102.

15. Y. Bistriz, $Z$-domain continued fraction expansions for stable discrete system polynomials, IEEE Trans. Circuits Syst. 32 (1985) 1162-1166.

16. M. Ismail, New z-domain continued fraction expansions, IEEE Trans. Circuits Syst. 32 (1985) 754-758.

17. X. Nie and R. Unbehauen, A novel efficient algorithm for stability test by continued fraction expansion with application to 2-D digital filters, IEEE Trans. Circuits Syst. 36 (1989) 315-317. 